\title{
EDITORIAL
}

\section{Maintenance of Certification: The Profes- sion's Response to Physician Quality}

\author{
Paul V. Miles, MD \\ American Board of Pediatrics, Chapel Hill, North Carolina
}

Ann Fam Med 2011;9:196-197. doi:10.1370/afm.1254

M aintenance of certification (MOC) represents a profound change in the approach by the medical profession to assessing physician quality. MOC acknowledges that current relevant medical knowledge is necessary, but not sufficient, and that other core competencies are necessary for physicians to deliver high-quality care. For the past 10 years the American Board of Medical Specialties (ABMS) boards have been designing and implementing MOC programs with the goal of helping physicians continuously improve professional development and clinical performance. Competency in quality improvement as evidenced by the ability to measure and systematically improve care is a key component of MOC.

The article by Xierli et $\mathrm{al}^{1}$ reports on the American Board of Family Medicine's (ABFM) evaluation of the rate and demographics of diplomate participation in the initial version of maintenance of certification for family medicine (MC-FM) for the first 7-year cycle from 2003 to 2010. In quality improvement terms, the ABFM has been conducting a series of Plan-DoStudy-Act (PDSA) cycles, and this report represents the study phase.

There are several important observations. The first is that for a process change such as MOC (completion of multiple self-assessment modules or performance in practice modules) to have an effect, there has to be high reliability or compliance with the proposed

Conflicts of interest: Dr Miles is a member of the American Board of Family Medicine Foundation.

\section{CORRESPONDING ADDRESS}

Paul V. Miles, MD

Senior Vice President for Quality and Maintenance of Certification The American Board of Pediatrics

111 Silver Cedar Ct

Chapel Hill, NC 27514

PMiles@abpeds.org changes. Certification and MOC in family medicine both show high rates of participation, with $85 \%$ of family physicians being board certified and $91 \%$ of eligible diplomates participating in MC-FM. This degree of voluntary participation in a process that requires investments of time and money implies that family physicians value board certification and MC-FM as a professional activity developed by their peers. To the degree that MC-FM requires a change in physician behavior, there are few other examples of programs that can match this level of compliance.

At a time when there is widespread interest in how to change physician practice, MOC appears to be a strong potential driver for change. But there is room for improvement. This study documents considerable variation in participation in both the initial certification process and MC-FM. Older male family physicians in solo practice in poor or underserved urban areas are at the greatest risk of having a lapsed certificate or not fully participating in MC-FM. A recent article in the New York Times ${ }^{2}$ highlights a growing concern with the quality of care of older physicians. This study suggests that family physicians aged more than 55 years are twice as likely as their younger colleagues to have allowed their certification to lapse and are less likely to participate in MC-FM. The authors also appropriately call attention to the potential impact of a lower rate of MC-FM participation on quality of care for poor and underserved patients.

From a quality improvement perspective, each physician that does not maintain certification can be viewed as a defect in the system, and the ABFM has begun the process of root cause analysis to try to understand the causes of lack of participation. Some differences, such as greater participation by physicians in group practice compared with physicians in solo practice and greater participation by family physicians who teach residents or fellows, suggest ready explanations, but others, such as significantly greater partici- 
pation by women family physicians, are less clear. The priority areas of focus appear to be older physicians, organizational support or a culture that supports participation, and practices' patient and community demographics. The aim is to identify changes that can be tested to improve participation in subsequent cycles.

This study is an important first step. But not all change is improvement. This work documents success around a process measure-participation in the new MC-FM-and identifies opportunities for focused interventions to improve participation. The next step is to demonstrate improvement in outcomes. Does participation in MC-FM lead to sustained changes in physician behavior, professional development, and the ability to improve patient outcomes? Is MOC helping close the gaps in quality? Data are accumulating that periodic assessment of medical knowledge is associated with higher quality of care. ${ }^{3}$ Preliminary analysis of MOC-approved quality improvement activities suggests that participation can lead to improved outcomes for both Web-based modules, such as performance in practice modules, and for rigorously designed quality improvement projects. ${ }^{3}$ In 2010 , as part of the American Board of Pediatrics MOC program, 2,731 pediatricians completed a hand hygiene Web-based module involving more than 80,000 patient encounters at baseline and another 80,000 plus encounters after the first quality improvement cycle. There was a $10 \%$ improvement in hand hygiene rates from $80 \%$ to $90 \%$ (unaudited patient or observer reported data). Two published qualtiy improvement studies by Miller et al and Mandel and Kotagal, which have been approved for MOC Part 4 credit, have shown improved outcomes of care and reduced costs in pediatric intensive care unit catheter-associated blood stream infections and outpatient asthma care, respectively., ${ }^{4,5}$

This study shows that physicians will participate in MOC. The challenge for the boards is to develop activities that are potentially relevant and effective and to test them through multiple MOC PDSA cycles. $\mathrm{MOC}$ represents the profession's response to the issue of physician quality. MOC presents an opportunity for the boards to be drivers of change in creating a culture for quality improvement and increasing physician competency in improvement science. It is important to make sure it works.

To read or post commentaries in response to this article, see it online at http://www.annfammed.org/cgi/content/full/9/3/196.

Key words: Certification; education, continuing; maintenance of certification; health services research; quality of health care; health policy research

Submitted April 7, 2011; accepted April 8, 2011.

\section{References}

1. Xierali IM, Rinaldo JCB, Green LA, et al. Family physician participation in maintenance of certification. Ann Fam Med. 2011;9(3): 203-210.

2. As doctors age, worries about their ability grow. NY Times. Jan 24, 2011.

3. Holmboe ES, Wang Y, Meehan TP, et al. Association between maintenance of certification examination scores and quality of care for medicare beneficiaries. Arch Intern Med. 2008;168(13):1396-1403.

4. Miller MR, Griswold M, Harris JM 2nd, et al. Decreasing PICU catheter-associated bloodstream infections: NACHRI's quality transformation efforts. Pediatrics. 2010;125(2):206-213

5. Mandel KE, Kotagal UR. Pay for performance alone cannot drive quality. Arch Pediatr Adolesc Med. 2007;161(7):650-655. 